\title{
Macroscopic Degeneracy of Zero-Mode Rotating Surface States in 3D Dirac and Weyl Semimetals under Radiation
}

\begin{abstract}
José González and Rafael A. Molina
Instituto de Estructura de la Materia, IEM-CSIC, Serrano 123, Madrid 28006, Spain

(Received 18 December 2015; revised manuscript received 30 March 2016; published 15 April 2016)

We investigate the development of novel surface states when 3D Dirac or Weyl semimetals are placed under circularly polarized electromagnetic radiation. We find that the hybridization between inverted Floquet bands opens, in general, a gap, which closes at so-called exceptional points found for complex values of the momentum. This corresponds to the appearance of midgap surface states in the form of evanescent waves decaying from the surface exposed to the radiation. We observe a phenomenon reminiscent of Landau quantization by which the midgap surface states get a large degeneracy proportional to the radiation flux traversing the surface of the semimetal. We show that all of these surface states carry angular current, leading to an angular modulation of their charge that rotates with the same frequency of the radiation, which should manifest in the observation of a macroscopic chiral current in the irradiated surface.
\end{abstract}

DOI: 10.1103/PhysRevLett.116.156803

Introduction.-In recent years we have witnessed the discovery of several types of materials characterized by having electron quasiparticles with linear momentum dispersion. Graphene was certainly the first of those materials [1], but afterwards we learned about the topological insulators [2,3], to end up more recently with the investigation of 3D semimetals whose low-energy excitations behave as Dirac [4-6] or Weyl fermions [7,8].

These materials have attracted a lot of attention for their potential to develop a new type of electronic transport without dissipation. The key idea is that of topological protection, which has its precedent in the edge states of the quantum Hall effect. The surface states in the novel materials may also have a well-defined chirality, protecting them against backscattering. Both properties are fundamental for revolutionary applications in spintronics and fault-tolerant quantum computation [2,3].

In this search, the interaction between light and matter can play an important role, since the electromagnetic radiation may be a versatile resource to change and control topological states of matter. In 2D semimetals, it can open a gap in the bulk, leading to chiral currents at the boundary of the electron system [9-18], as observed on the surface of 3D topological insulators [19]. The effect of the radiation has also been investigated in the case of 3D Dirac and Weyl semimetals, focusing on bulk properties [20-22].

In this Letter, we investigate the development of novel surface states when a 3D Dirac or Weyl semimetal is placed under circularly polarized electromagnetic radiation. We will show that such states are intimately related to avoided crossings at the gap that opens up from the hybridization of inverted Floquet bands, as represented in Fig. 1. The gap closes at so-called exceptional points (EPs) [23,24], which are celebrated in the context of non-Hermitian Hamiltonians and appear here for complex values of the momentum describing the evanescence into the 3D semimetal. The stability of the novel surface states is then guaranteed by a new mechanism of topological protection, relying on the fact that each EP comes as a branch point in the spectrum which cannot be removed by small perturbations.

The genuine feature of the novel surface states is that they all carry a significant angular current, with the same chirality of the photon polarization and the frequency of the radiation. Such states may prove especially useful in the current drive towards increasing the frequency limits of electronic devices [25].

Model and Floquet theory.-A low-energy Hamiltonian for a 3D Dirac semimetal around the Brillouin zone center, considering terms up to quadratic order in the quasimomentum $\mathbf{k}$, can be written as $[26,27]$
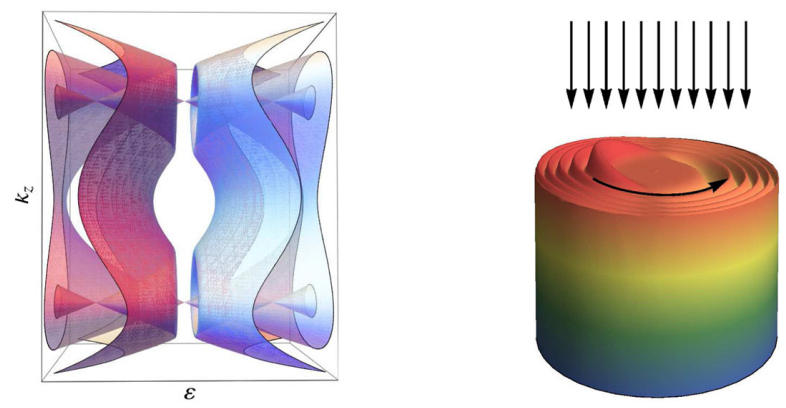

FIG. 1. Left: Schematic representation of the first Floquet bands with quasienergy $\epsilon$ and $\epsilon \pm \hbar \Omega$ ( $\Omega$ being the radiation frequency) for a model of 3D semimetal with two nodes along the momentum axis $k_{z}$ (red color denotes positive energy while light blue denotes negative energy, the direction transverse to $k_{z}$ represents additional components of the momentum). Right: Geometry of 3D semimetal with the surface exposed to the radiation where the evanescent states appear. 


$$
\begin{gathered}
H=\epsilon_{0}(\mathbf{k}) \llbracket+M(\mathbf{k}) \sigma_{z}+\hbar v\left(\zeta k_{x} \sigma_{x}-k_{y} \sigma_{y}\right), \\
\epsilon_{0}(\mathbf{k})=c_{0}+c_{1} k_{z}^{2}+c_{2}\left(k_{x}^{2}+k_{y}^{2}\right), \\
M(\mathbf{k})=m_{0}-m_{1} k_{z}^{2}-m_{2}\left(k_{x}^{2}+k_{y}^{2}\right),
\end{gathered}
$$

where $\square$ is the $2 \times 2$ identity matrix, $\sigma_{i}, i=x, y, z$ are the Pauli matrices, and $\zeta= \pm 1$ sets the chirality in the Dirac cones. The solution of the eigenvalue problem is

$$
E_{ \pm}=\epsilon_{0}(\mathbf{k}) \pm \sqrt{M(\mathbf{k})^{2}+\hbar^{2} v^{2}\left(k_{x}^{2}+k_{y}^{2}\right)} .
$$

With the parameters $m_{0}, m_{1}, m_{2}<0$ to reproduce band inversion, the spectrum shows Dirac crossings at $\mathbf{k}_{c}=$ $\left(0,0, \pm \sqrt{m_{0} / m_{1}}\right)$. Ignoring the part proportional to the unit matrix $\epsilon_{0}(\mathbf{k})$, we can expand the Hamiltonian linearly around each Dirac point to obtain a model for 3D massless Dirac fermions with anisotropic linear dispersion $E(\mathbf{k})= \pm \sqrt{\hbar^{2} v^{2}\left(k_{x}^{2}+k_{y}^{2}\right)+4 m_{0} m_{1}\left(k_{z}-k_{c, z}\right)^{2}}$.

Consider illumination by circularly polarized offresonant light of frequency $\Omega$ and field amplitude $\mathcal{F}$. In the case of polarization in the $x-y$ plane, light produces the vector potential in the dipolar approximation $\mathcal{A}(t)=$ $\mathcal{A}(\eta \sin \Omega t, \cos \Omega t, 0)$, where $\eta= \pm 1$ for right and left circularly polarized beams, respectively, and $\mathcal{A}=\mathcal{F} / \Omega$. We make the Peierls substitution $\mathbf{k} \rightarrow \mathbf{k}+\mathcal{A}(t)$ and use Floquet theory in order to compute the band structure in the presence of the radiation field [28]. Solutions of the time-dependent Schrödinger equation in the case of time-periodic Hamiltonians have the form $|\Psi(t)\rangle=$ $e^{-i \epsilon t / \hbar}|\Phi(t)\rangle$, with a conserved quantity, the quasienergy $\epsilon$, playing a similar role to the energy in the timeindependent Schödinger equation. The Floquet states $|\Phi(t)\rangle$ are periodic in time with the same period as the Hamiltonian and they can be developed in Fourier series, $|\Phi(t)\rangle=\sum_{m} e^{-i m \Omega t}\left|u_{\alpha}^{m}\right\rangle$. This transforms the timedependent Schrödinger equation into

$$
\sum_{n} H^{m n}\left|u_{\alpha}^{n}\right\rangle=\left(\epsilon_{\alpha}+m \hbar \Omega\right)\left|u_{\alpha}^{m}\right\rangle,
$$

where the Floquet Hamiltonian matrix elements are given by $H^{m n}=(1 / T) \int_{0}^{T} d t H(t) e^{i(m-n) \Omega t}, T=2 \pi / \Omega$ being the period of the time-dependent Hamiltonian [28].

As a relevant example, we perform calculations in an infinite wire in the $z$ direction with a finite square section in the $x-y$ plane. Using the Floquet formalism, we calculate the band structure as a function of $k_{z}$ discretizing the model Hamiltonian with a standard tight-binding regularization including the Floquet subbands. Results with parameters of the model in the topological Dirac semimetal regime are shown in Fig. 2, where we compare the energy of the bulk bands (left) with the quasienergy of the bands in the wire (right) as a function of $k_{z}$. The structure of the bands
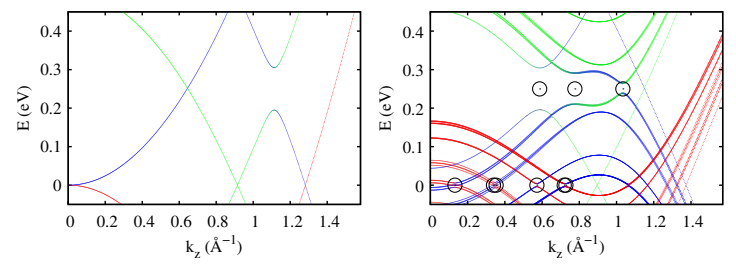

FIG. 2. Band structure for model parameters $c_{0}=c_{1}=c_{2}=$ $0.0, \quad m_{0}=-0.5 \mathrm{eV}, \quad m_{1}=-0.605 \mathrm{eV} \AA^{2}, \quad m_{2}=-1.0 \mathrm{eV} \AA^{2}$, $\hbar v=1.1 \mathrm{eV} \AA, \hbar \Omega=0.5 \mathrm{eV}, \mathcal{A}=0.05 \AA^{-1}$, in the bulk (left-hand panel) and for a wire of section $S=150 \times 150 \AA^{2}$ in the $x-y$ plane (right-hand panel). The position of the Dirac cone at zero field is $k_{c, z}=\sqrt{m_{0} / m_{1}}=0.909 \AA^{-1}$. The color represents the predominant Floquet mode of the particular state in a RGB coding (RGB standing for red-green-blue) where the intensity of each mode is mapped to the intensity of each fundamental color; $n=-1,0,1$ correspond to red, green, and blue, respectively. The position of the evanescent waves obtained as strictly real solutions of the eigenvalue problem after including an imaginary part in $k_{z}$ of $\operatorname{Im}\left(k_{z}\right)=0.01 \AA^{-1}$ is shown with black circles with a dot. A few high-energy bands with $n=-1$ index not affecting the avoided crossing structures have been erased for clarity of presentation.

wrapping up in the Floquet-Brillouin zone is very apparent. In the middle of the Floquet-Brillouin zone at $\epsilon=0$, a gap opens between states with Floquet modes $n=-1$ (red) and $n=1$ (blue), the hybridization being a second order effect leading to a quite small gap, proportional to $\mathcal{A}^{2}$. The much bigger gap (proportional to $\mathcal{A}$ ) between modes $n=0$ (green) and $n=1$ is in one of the edges of the FloquetBrillouin zone at $\epsilon=\hbar \Omega / 2$, with a mirror structure (not shown) at $\epsilon=-\hbar \Omega / 2$ between modes $n=0$ and $n=-1$.

In order to explore the possibility of evanescent states in the $z$ direction, we solve the Schrödinger equation in the same geometry but including a finite imaginary part in $k_{z}$. The Hamiltonian becomes then non-Hermitian and the eigenenergies acquire a finite imaginary part. These are obviously nonphysical solutions of the problem. However, non-Hermitian Hamiltonians may have eigenenergies that are strictly real, in the form of EPs [23,24]. These correspond to physical evanescent solutions with the imaginary part of the momentum giving the decay length of a wave in a semi-infinite geometry. The black circles in Fig. 2 mark the presence of the evanescent states in the gaps induced by the radiation. Not all of the avoided crossings present evanescent states, that depends on the properties of the actual bands involved. However, the number of avoided crossings is proportional to $\mathcal{A}$ and to the area of the section of the wire. This may imply, in general, a large degeneracy of evanescent states, whose origin is clarified in the continuum limit discussed in what follows.

Quantization and localization of zero-mode surface states.-In order to unveil the properties of the novel surface states, we focus now on the low-energy physics around any of the nodes of the Hamiltonian Eq. (1), taking, 
moreover, the projection for a given chirality $\zeta$. This amounts to linearizing $M(\mathbf{k})$ about the node at $\mathbf{k}=\mathbf{k}_{c}$, which leads in real space to the Hamiltonian for a single Weyl quasiparticle:

$$
\begin{aligned}
H_{W}= & -i \hbar\left(v \sigma_{x} \partial_{x}+v \sigma_{y} \partial_{y}+v_{z} \sigma_{z} \partial_{z}\right) \\
& +\hbar v \mathcal{A}\left[\sigma_{x} \cos (\Omega t)+\sigma_{y} \sin (\Omega t)\right],
\end{aligned}
$$

with a velocity $v_{z} \neq v$ along the $z$ axis [29].

$H_{W}$ can be translated into a time-independent Hamiltonian after applying the unitary transformation $U=e^{-i J_{z} \Omega t / \hbar}$, with the projection of the total angular momentum $J_{z}=-i \hbar x \partial_{y}+i \hbar y \partial_{x}+\hbar \sigma_{z} / 2 \quad$ [30]. This leads to the transformed Hamiltonian

$$
\begin{aligned}
\tilde{H}_{W} & =U^{\dagger} H_{W} U-i \hbar U^{\dagger} \partial_{t} U \\
& =-i \hbar\left(v \sigma_{x} \partial_{x}+v \sigma_{y} \partial_{y}+v_{z} \sigma_{z} \partial_{z}\right)-\Omega J_{z}+\hbar v \mathcal{A} \sigma_{x} .
\end{aligned}
$$

Each eigenvector $\chi$ of Eq. (7) corresponds then to a solution $\Psi(t)$ of the original time-dependent Schrödinger equation, given by $\Psi(t)=e^{-i J_{z} \Omega t / \hbar} e^{-i \varepsilon t / \hbar} \chi$ [ $\varepsilon$ being the eigenvalue of Eq. (7)]. This shows that the eigenvalues $j_{z}$ of the projection $J_{z}$ can be used in this approach to label the different sidebands arising from the irradiation.

After applying the gauge transformation $P=$ $\exp (-i \mathcal{A} x)$ to Eq. (7), the Hamiltonian becomes [in polar coordinates $r, \theta$ and with $\left.\sigma_{ \pm}=\left(\sigma_{x} \pm i \sigma_{y}\right) / 2\right]$

$$
\begin{aligned}
\tilde{H}_{W}^{\prime}= & -i \hbar v \sum_{s= \pm} e^{-s i \theta}\left(\partial_{r}-s i \frac{1}{r} \partial_{\theta}\right) \sigma_{s} \\
& -i \hbar v_{z} \sigma_{z} \partial_{z}-\hbar \Omega\left(-i \partial_{\theta}+\frac{\sigma_{z}}{2}\right)-\hbar \Omega \mathcal{A} r \sin (\theta) .
\end{aligned}
$$

The last term in Eq. (8) is responsible for the coupling between states with different angular momenta $j_{z}$. Then, the avoided crossing of the bands with $j_{z}= \pm \hbar / 2$ gives rise to a gap around zero energy in the spectrum of Eq. (8). This point actually corresponds to a quasienergy $\epsilon=\hbar \Omega / 2$ in the conventional Floquet approach, since $U$ has the effect of shifting the energy of the sidebands in the present approach by half-integer multiples of $\hbar \Omega$.

The development of the gap can be captured (for not too large amplitude $\mathcal{A}$ ) by solving in the space spanned by a linear combination of spinors with projection of the total angular momentum $j_{z}= \pm \hbar / 2$,

$$
\chi=\left(\begin{array}{c}
\phi_{1}(r) \\
e^{i \theta} \phi_{2}(r)
\end{array}\right) e^{i k_{z} z}+\left(\begin{array}{c}
e^{-i \theta} \phi_{3}(r) \\
\phi_{4}(r)
\end{array}\right) e^{i k_{z} z}
$$

We have considered specifically a cylindrical geometry with the section at $z=0$ exposed to the radiation, as represented in Fig. 1 [31]. A typical shape of the gap is

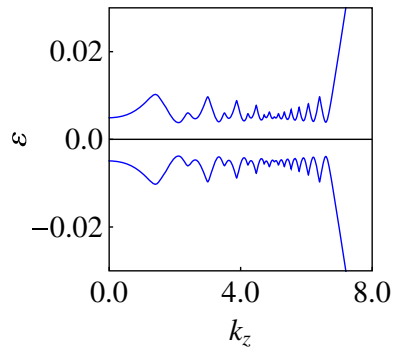

(a)

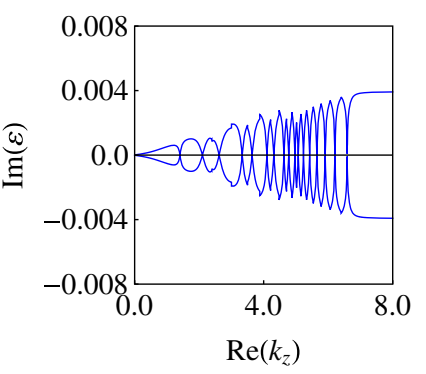

(b)
FIG. 3. (a) Plot of the gap of the Hamiltonian Eq. (8) (in eV) as a function of real $k_{z}$ (measured in units of the inverse of the typical microscopic length scale $a$ in the material) for $\hbar v / a=1.1 \mathrm{eV}, \hbar \Omega=0.5 \mathrm{eV}, \mathcal{A}=0.005 a^{-1}$, and radius $R=$ $200 a$ of the cylindrical geometry considered in the text. (b) Imaginary part of the lowest eigenvalue of the Hamiltonian Eq. (8) (in $\mathrm{eV})$ as a function of $\operatorname{Re}\left(k_{z}\right)$, for complex momenta with $\operatorname{Im}\left(k_{z}\right)=$ $0.08 a^{-1}$ and the same parameters as in (a).

shown in Fig. 3(a), where we see that it has several oscillations before recovering the linear dispersion of the original cones beyond a certain $k_{z}$. This is indeed the generic behavior, with an increasing number of oscillations as the radius $R$ of the cylinder grows.

The origin of the oscillations in the gap is clarified by noting that they arise from the existence of EPs in the spectrum of the Hamiltonian Eq. (8), corresponding to values of $k_{z}$ inside the complex plane where the gap closes. Around each oscillation of the gap, the lowest eigenvalue $\varepsilon$ behaves as a function of complex $k_{z}$ as $\varepsilon \sim \sqrt{k_{i}-k_{z}} \sqrt{\bar{k}_{i}-k_{z}}$, with EPs at complex conjugate momenta $k_{i}, \bar{k}_{i}$ [28]. Evanescent states with $\operatorname{Im}(\varepsilon)=0$ exist then in the segment $\left|\operatorname{Im}\left(k_{z}\right)\right| \leq\left|\operatorname{Im}\left(k_{i}\right)\right|$, for each pair $k_{i}, \bar{k}_{i}$. We have represented, for instance, in Fig. 3(b) the behavior of the imaginary part of the lowest eigenvalue, when $\operatorname{Im}\left(k_{z}\right) \neq 0$, as a function of the real part of $k_{z}$. We observe the recurrent development of complex momenta at which the eigenvalue becomes purely real, leading to a set of evanescent eigenstates in perfect correspondence with the minima of the gap in Fig. 3(a).

We find then that the evanescent states are preserved by a mechanism of topological protection, as the branch cuts cannot be undone in the complex plane unless the branch points coalesce in pairs. It can be seen that the imaginary part of the EPs has a very smooth dependence on the frequency $\Omega$, while it grows linearly with the amplitude $\mathcal{A}$ [32]. The number of zero-mode evanescent states may be actually characterized from the number of branch points that the lowest band $\varepsilon\left(k_{z}\right)$ has in the complex plane.

It can be checked that the number of EPs increases as the radius $R$ grows, leading to a definite pattern of quantization in the surface of our geometry. It can be seen that the order of each zero in the plot of Fig. 3(b) (from right to left) also gives the extent of the localization that the corresponding evanescent wave has in the radial direction, as illustrated in 

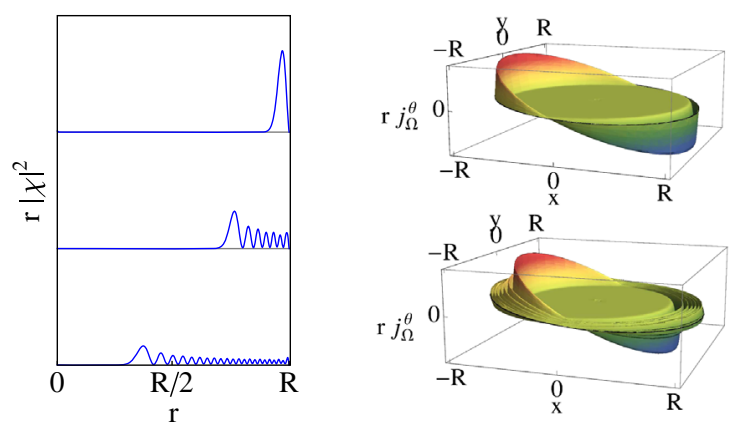

FIG. 4. Left: Probability distribution $r|\chi|^{2}$ along the radial direction for evanescent states corresponding to three decreasing values of $\operatorname{Re}\left(k_{z}\right)$ with $\operatorname{Im}(\varepsilon)=0$ (starting with the outermost evanescent state) for the same parameters as in Fig. 3 and $R=400 a$. Right: Time-dependent angular current $r j_{\Omega}^{\theta}$ for the states in the upper part (top) and the middle part (bottom) of the left-hand panel, represented for fixed time $t=0$ at the irradiated surface of the cylindrical geometry.

Fig. 4. The peak in the probability distribution shifts to lower values of $r$ as $\operatorname{Re}\left(k_{z}\right)$ decreases, eventually reaching the innermost region of the surface. The number of evanescent states, besides growing linearly with the frequency $\Omega$ and the amplitude $\mathcal{A}$, actually becomes proportional to the area of the irradiated surface, at a rate of roughly one state per $(100 a)^{2}$ (for $\hbar \Omega=0.1 \mathrm{eV}$, $\mathcal{A}=0.01 a^{-1}, a$ being a typical microscopic length scale in the material).

Furthermore, the midgap evanescent states evolve in time with a rotation of their charge along the angular variable $\theta$. This can be shown by computing the angular component $j^{\theta}$ of the probability current for the Hamiltonian Eq. (6). This current has a static contribution, $j_{\text {static }}^{\theta}=$ $-i(v / r)\left(\phi_{1}^{*} \phi_{2}+\phi_{3}^{*} \phi_{4}\right)+$ H.c., which leads to a very small intensity when integrated over the radial direction, as a consequence of the tendency of the two contributions from $j_{z}= \pm \hbar / 2$ states to cancel each other [28]. For $\hbar \Omega=$ $0.5 \mathrm{eV}$ and $\mathcal{A}=0.005 a^{-1}$, we get, for instance, values of the intensity $\int d r r j_{\text {static }}^{\theta}$ between $\sim 10^{-5} v / a$ and $\sim 10^{-4} v / a$, when computing for evanescent states from the outermost to the innermost region of the surface. However, there is also a non-negligible time-dependent contribution to $j^{\theta}$ [28], given by

$j_{\Omega}^{\theta}=-i(v / r)\left(\phi_{1}^{*} \phi_{4} e^{-i(\theta-\Omega t)}+\phi_{3}^{*} \phi_{2} e^{i(\theta-\Omega t)}\right)+$ H.c.,

which has a periodic dependence on $\theta-\Omega t$, as shown in Fig. 4 [33]. The intensity corresponding to $j_{\Omega}^{\theta}$ has maxima (in the angular variable) which turn out to be, in general, about 2 orders of magnitude above the intensity obtained from $j_{\text {static }}^{\theta}$, for every evanescent state [28].

When introduced in the continuity equation, the form of $j_{\Omega}^{\theta}$ implies that the charge of each state must have a periodic modulation in the angular variable, and that it must rotate with frequency $\Omega$ along the concentric rings where each evanescent state is confined [28]. From a practical point of view, this leads to the formation of rotating dipoles on the surface of the system, whose movement can be controlled by tuning the parameters of the radiation.

While we have referred here to the hybridization of states with $j_{z}= \pm \hbar / 2$, the coupling of sidebands with higher values of $J_{z}$ results in surface states with similar properties of localization and time evolution [28]. It can be seen, in particular, that the hybridization of sidebands with values of $j_{z}$ differing by $2 \hbar$ leads also to evanescent states, which correspond in that case to the EPs found at $\epsilon=0$ in the preceding section.

Conclusion.-An important practical consideration in this study is that the infrared radiation may penetrate sufficiently deep into the 3D semimetals, given the limited screening in these materials. The penetration length $l$ can be estimated as the inverse of the absorption coefficient $\alpha$, which is expressed in terms of the dielectric function $\epsilon(\Omega)$ as $\alpha=2 \Omega \operatorname{Im} \sqrt{\epsilon(\Omega)} / c$. We find, for instance, that $l \sim$ $1 \mu \mathrm{m}$ in the near infrared ( $\Omega \sim 100 \mathrm{THz})$ [28], which is a large enough distance to afford the development of the evanescent states.

The magnitude of the component $j_{\Omega}^{\theta}$ of the current offers good perspectives to observe experimentally the novel surface states. In our cylindrical geometry, the intensity of the current across the radial direction, $I=e \int d r r j_{\Omega}^{\theta}$, gets maxima (in the angular variable) that range between $\sim 10^{-1}$ and $\sim 1 \mu \mathrm{A}$, for individual states taken from the outermost to the innermost region in the top surface. The total intensity could be enhanced by a large additional factor, in a device able to measure the contribution of a significant part of the surface states.

A suitable experimental setup (i.e., two electrodes on top of the surface of the semimetal) may be able to convert the rotation of the charge in the surface states into an electrical current, if the device is made to work as a rectenna. This may benefit from recent developments that make it possible to rectify currents oscillating even at the frequency of visible light [34]. In our case, it may greatly help the fact that the rotation of all the surface states is synchronized with that of the radiation fields, making easier the observation of the macroscopic chiral current that may develop at the irradiated surface.

We acknowledge financial support from MICINN (Spain) through Grant No. FIS2011-23713 and MINECO (Spain) through Grants No. FIS2012-34479 and No. FIS2014-57432-P.

[1] K. S. Novoselov, A. K. Geim, S. V. Morozov, D. Jiang, Y. Zhang, S. V. Dubonos, I. V. Grigorieva, and A. A. Firsov, Electric field effect in atomically thin carbon films, Science 306, 666 (2004). 
[2] M. Z. Hasan and C. L. Kane, Topological insulators, Rev. Mod. Phys. 82, 3045 (2010).

[3] X.-L. Qi and S.-C. Zhang, Topological insulators and superconductors, Rev. Mod. Phys. 83, 1057 (2011).

[4] Z. K. Liu, B. Zhou, Y. Zhang, Z. J. Wang, H. M. Weng, D. Prabhakaran, S.-K. Mo, Z. X. Shen, Z. Fang, X. Dai, Z. Hussain, and Y. L. Chen, Discovery of a three-dimensional topological Dirac semimetal, $\mathrm{Na}_{3} \mathrm{Bi}$, Science 343, 864 (2014).

[5] M. Neupane, S. Xu, R. Sankar, N. Alidoust, G. Bian, C. Liu, I. Belopolski, T.-R. Chang, H.-T. Jeng, H. Lin, A. Bansil, F. Chou, and M. Z. Hasan, Observation of a three-dimensional topological Dirac semimetal phase in high-mobility $\mathrm{Cd}_{3} \mathrm{As}_{2}$, Nat. Commun. 5, 3786 (2014).

[6] S. Borisenko, Q. Gibson, D. Evtushinsky, V. Zabolotnyy, B. Büchner, and R. J. Cava, Experimental Realization of a Three-Dimensional Dirac Semimetal, Phys. Rev. Lett. 113, 027603 (2014).

[7] S.-Y. Xu, I. Belopolski, N. Alidoust, M. Neupane, G. Bian, C. Zhang, R. Sankar, G. Chang, Z. Yuan, C.-C. Lee, S.-M. Huang, H. Zheng, J. Ma, D. S. Sanchez, B. Wang, A. Bansil, F. Chou, P. P. Shibayev, H. Lin, S. Jia, and M. Z. Hasan, Discovery of a Weyl fermion semimetal and topological Fermi arcs, Science 349, 613 (2015).

[8] B. Q. Lv, H. M. Weng, B. B. Fu, X. P. Wang, H. Miao, J. Ma, P. Richard, X. C. Huang, L. X. Zhao, G. F. Chen, Z. Fang, X. Dai, T. Qian, and H. Ding, Experimental Discovery of Weyl Semimetal TaAs, Phys. Rev. X 5, 031013 (2015).

[9] T. Oka and H. Aoki, Photovoltaic Hall effect in graphene, Phys. Rev. B 79, 081406 (2009).

[10] N. H. Lindner, G. Refael, and V. Galitski, Floquet topological insulators, Nat. Phys. 7, 490 (2011).

[11] J.-I. Inoue and A. Tanaka, Photoinduced Transition between Conventional and Topological Insulators in Two-Dimensional Electronic Systems, Phys. Rev. Lett. 105, 017401 (2010).

[12] T. Kitagawa, E. Berg, M. Rudner, and E. Demler, Topological characterization of periodically driven quantum systems, Phys. Rev. B 82, 235114 (2010).

[13] T. Kitagawa, T. Oka, A. Brataas, L. Fu, and E. Demler, Transport properties of nonequilibrium systems under the application of light: Photoinduced quantum Hall insulators without Landau levels, Phys. Rev. B 84, 235108 (2011).

[14] Z. Gu, H. A. Fertig, D. P. Arovas, and A. Auerbach, Floquet Spectrum and Transport through an Irradiated Graphene Ribbon, Phys. Rev. Lett. 107, 216601 (2011).

[15] H. L. Calvo, H. M. Pastawski, S. Roche, and L. E. F. Foa Torres, Tuning laser-induced band gaps in graphene, Appl. Phys. Lett. 98, 232103 (2011).

[16] A. Gómez-León and G. Platero, Floquet-Bloch Theory and Topology in Periodically Driven Lattices, Phys. Rev. Lett. 110, 200403 (2013).

[17] P. M. Perez-Piskunow, G. Usaj, C. A. Balseiro, and L. E. F. Foa Torres, Floquet chiral edge states in graphene, Phys. Rev. B 89, 121401(R) (2014).

[18] H. L. Calvo, L. E. F. Foa Toores, P. M. Perez-Piskiunow, C. A. Balseiro, and G. Usaj, Floquet interface states in illuminated three dimensional topological insulators, Phys. Rev. B 91, 241404(R) (2015).

[19] Y. H. Wang, H. Steinberg, P. Jarillo-Herrero, and N. Gedik, Observation of Floquet-Bloch states on the surface of a topological insulator, Science 342, 453 (2013).

[20] R. Wang, B. Wang, R. Shen, L. Sheng, and D. Y. Xing, Floquet Weyl semimetal induced by off-resonant light, Europhys. Lett. 105, 17004 (2014).

[21] A. Narayan, Floquet dynamics in two-dimensional semiDirac semimetals and three-dimensional Dirac semimetals, Phys. Rev. B 91, 205445 (2015).

[22] C.-K. Chan, P. A. Lee, K. S. Burch, J. H. Han, and Y. Ran, When Chiral Photons Meet Chiral Fermions: Photoinduced Anomalous Hall Effects in Weyl Semimetals, Phys. Rev. Lett. 116, 026805 (2016).

[23] M. V. Berry, Physics of nonhermitian degeneracies, Czech. J. Phys. 54, 1039 (2004).

[24] W. D. Heiss, The physics of exceptional points, J. Phys. A 45, 444016 (2012).

[25] F. Krausz and M. I. Stockman, Attosecond metrology: from electron capture to future signal processing, Nat. Photonics 8, 205 (2014).

[26] Z. Wang, Y. Sun, X.-W. Chen, C. Franchini, G. Xu, H. Weng, X. Dai, and Z. Fang, Dirac semimetal and topological phase transitions in $A_{3} \mathrm{Bi}(A=\mathrm{Na}, \mathrm{K}, \mathrm{Rb})$, Phys. Rev. B 85, 195320 (2012).

[27] Z. Wang, H. Weng, Q. Wu, X. Dai, and Z. Fang, Threedimensional Dirac semimetal and quantum transport in $\mathrm{Cd}_{3} \mathrm{As}_{2}$, Phys. Rev. B 88, 125427 (2013).

[28] See Supplemental Material at http://link.aps.org/ supplemental/10.1103/PhysRevLett.116.156803 for the estimate of the penetration of the radiation, the description of the Floquet approach, the signatures of the exceptional points, and the evaluation of the current for the evanescent states.

[29] We set in what follows $v_{z}=0.05 v$, taking as a guide the relative magnitudes observed in real $3 \mathrm{D}$ semimetals.

[30] A. López, A. Scholz, B. Santos, and J. Schliemann, Photoinduced pseudospin effects in silicene beyond the off-resonant condition, Phys. Rev. B 91, 125105 (2015).

[31] We note that, in the semi-infinite geometry, two modes with momenta $k_{z}$ and $-k_{z}$ are to be superposed, in order to build each eigenstate vanishing at the top boundary of the cylinder.

[32] A typical magnitude of the imaginary part of the EPs is, for instance, $\operatorname{Im}\left(k_{i}\right) \sim 0.1 a^{-1}$, for $\hbar \Omega=0.5 \mathrm{eV}$ and $\mathcal{A}=0.005 a^{-1}$, where $a$ stands for a typical microscopic length scale in the material.

[33] Our definition of the current in Eq. (10) (as well as that of $j_{\text {static }}^{\theta}$ ) omits for simplicity its decay along the $z$ axis, which is inherited from the evanescence of the surface states.

[34] A. Sharma, V. Singh, T. L. Bougher, and B. A. Cola, A carbon nanotube optical rectenna, Nat. Nanotechnol. 10, 1027 (2015). 